CCNY-HEP-06/5

April 2006

\title{
The Chern-Simons One-form and Gravity on a Fuzzy Space
}

\author{
V. P. NAIR \\ Physics Department \\ City College of the CUNY \\ New York, NY 10031 \\ E-mail: vpn@sci.ccny.cuny.edu
}

\begin{abstract}
The one-dimensional $\mathcal{N} \times \mathcal{N}$-matrix Chern-Simons action is given, for large $\mathcal{N}$ and for slowly varying fields, by the $(2 k+1)$-dimensional Chern-Simons action $S_{C S}$, where the gauge fields in $S_{C S}$ parametrize the different ways in which the large $\mathcal{N}$ limit can be taken. Since some of these gauge fields correspond to the isometries of the space, we argue that gravity on fuzzy spaces can be described by the one-dimensional matrix Chern-Simons action at finite $\mathcal{N}$ and by the higher dimensional Chern-Simons action when the fuzzy space is approximated by a continuous manifold.
\end{abstract}




\section{Introduction}

Noncommutative and fuzzy spaces have been interesting objects of research for a long time [1]. Such spaces can be realized as solutions, for example, branes, sometimes with specific background fields, in string theory and in the matrix version of $M$-theory [2. Gauge theories on such spaces are interesting since they can describe fluctuations of the brane solutions. Fuzzy spaces are noncommutative spaces which can be described by finite dimensional matrices. Examples of many fuzzy spaces, such as $S_{F}^{2}$, $\mathbf{C P}_{F}^{k}$, etc., have been constructed. When the dimension of the matrices which represent the coordinates becomes large, these spaces tend to their smooth counterparts $S^{2}, \mathbf{C P}^{k}$, etc. The finite dimensionality of the matrices means that there are only a finite number of modes for any fields defined on a fuzzy space. Therefore we may regard such theories as finite-mode approximations to the usual field theories on a smooth (and commutative) manifold [3]. It is thus an alternative method of regularization, analogous to the lattice regularization. It is also possible to preserve more symmetries in a fuzzification, compared to latticization ${ }^{1}$. The matrix description of branes can also be viewed as a regularization of the brane itself, and in this sense, it has become part of the standard repertoire of methods for analyzing branes [5]. While, for most theories, a new regulator may not mean very much, gravity is one case where fuzzy spaces could do significantly better, due to the possibility that fuzzification can preserve symmetries. Naturally there have been many investigations into gravity on fuzzy spaces, and more generally, on noncommutative spaces [6, 7]. Clearly, it is important to explore gravity on fuzzy spaces.

One can even go a bit further in these considerations, raising the question whether it is possible to describe the world by a finite-dimensional Hilbert space. Since there are many spaces, such as de Sitter space, which have finite entropy, a finite-dimensional Hilbert space describing all physical degrees of freedom is worthy of consideration, not just as a regulator, but as a basic premise for physical theories.

Fuzzy spaces also have an obvious connection to the lowest Landau level of a quantum Hall system [8]. This is most easily seen with a specific example, say, $\mathbf{C P}^{k}=S U(k+1) / U(k)$. The isometries of this space are given by $S U(k+1)$, with $U(k)$ as the local isotropy group, the analog of the Lorentz group for Minkowski space. The Riemann curvature has values in the Lie algebra of $U(k) \sim S U(k) \times U(1)$, and has constant components in the tangent frame basis. To formulate the Landau problem, consider $\mathbf{C P}^{k}$ with an additional constant "magnetic field" proportional to, say, the $U(1)$-component of the Riemann tensor. The lowest Landau level can then be characterized by a basis of wave functions which are, evidently,

\footnotetext{
${ }^{1}$ There may be other advantages to fuzzification as well. For example, it has been argued that inherent nonlocality of fuzzy spaces can be used to evade the fermion doubling problem of the lattice formulations in a way that still preserves most of the desirable symmetries [4].
} 
sections of a $U(1)$-bundle on $\mathbf{C P}^{k}$. The set of such lowest Landau level wave functions form a finite dimensional Hilbert space $\mathcal{H}_{N}$. Observables of the theory, for all dynamics projected to the lowest Landau level, are then arbitrary hermitian $(N \times N)$-matrices. The algebra of such observables is obviously $M_{a} t_{N}$, the matrix algebra of $(N \times N)$-matrices. With a suitable choice of a Laplacian $\Delta_{N}$, the triplet $\left(\mathcal{H}_{N}, M a t_{N}, \Delta_{N}\right)$ can then be taken as the definition of $\mathbf{C P}_{F}^{k}$, the fuzzy version of $\mathbf{C P}^{k}$. It is easy to show, by standard techniques of semiclassical analysis, that $\mathrm{Mat}_{N}$ tends to the algebra of functions on $\mathbf{C P}^{k}$ as $N$ becomes large.

The quantum Hall system thus gives us a model and a physical context to think about gravity on fuzzy spaces. (For a general discussion of various aspects of the quantum Hall system in higher dimensions, see references [9, 10, 11].) As for the dynamics, one can consider a Hall droplet of fermions for which the bulk and boundary dynamics have been analyzed in some detail [10. In what follows, it is the bulk dynamics which will be relevant. By virtue of the exclusion principle and the related incompressibility of the droplet, the bulk dynamics will be trivial, unless one introduces fluctuations in the gauge fields (or "magnetic fields"). Local changes in gauge fields can change the local density of states leading to rearrangements of fermions in the droplet, including changes in ddensity. Since such gauge fields can take values in $\underline{S U(k+1)}$ for $\mathbf{C} \mathbf{P}^{k}$, they can be interpreted as gauging the isometries of $\mathbf{C} \mathbf{P}^{k}$. Naturally, this suggests a way of introducing gravitational fields on a fuzzy space [12. ${ }^{2}$

There is one more important insight which can be elicited from this mapping of the fuzzy gravity problem to the quantum Hall system. Imagine we have a droplet of fermions of finite size in the lowest Landau level. The droplet may be characterized by a density matrix $\rho_{0}$. The general time evolution of the droplet is then given by a unitary tranformation $\rho_{0} \rightarrow U \rho_{0} U^{\dagger}$, obeying the standard quantum Liouville equation,

$$
i \frac{\partial \rho}{\partial t}=[K, \rho]
$$

where $K$ is the Hamiltonian. The action which leads to this equation is

$$
\begin{aligned}
S & =\int d t \operatorname{Tr} \rho_{0}\left(i U^{\dagger} \dot{U}-U^{\dagger} K U\right)=i \int d t \operatorname{Tr} \rho_{0}\left(U^{\dagger} D_{0} U\right) \\
D_{0} & =\frac{\partial}{\partial t}+A_{0}, \quad A_{0}=i K
\end{aligned}
$$

In reinterpreting the Hilbert space as defining a fuzzy space, the states correspond to points. This suggests that the dynamics of space itself (gravity) should be the same as the evolution of states in the quantum Hall image of it, and, hence, it is given by the action (2). This what the quantum Hall analogy gives us: The action (2), with some qualifications elaborated on

\footnotetext{
${ }^{2}$ It is also suggestive that quantum Hall droplets appear in the dual field theories for many gravitational backgrounds [13].
} 
below, should be the action for fuzzy gravity. Our basic principle is that the same equation for evolution should hold for the states corresponding to space as for states corresponding to matter. We shall now explore this in more detail, but need some technical results.

\section{The one-dimensional Chern-Simons action}

We will formulate the calculation of the effective action, which is the large $n$ limit of an action like (2), using $\mathbf{C P}^{k}=S U(k+1) / U(k)$ as the phase space. It is possible to choose other spaces for this purpose as well. Th final result we are aiming for will be independent of the specific choice. As mentioned before, constant magnetic fields on $\mathbf{C P}^{k}$ take values in $U(k)$, the Lie algebra of $U(k)$. Wave functions can be given in terms of a general $S U(k+1)$ element, say, a $(k+1) \times(k+1)$-matrix $g$. We will denote by $t_{a}, a=1,2, \cdots, k^{2}+2 k$, a set of hermitian matrices which form a basis for the Lie algebra of $S U(k+1)$ in the fundamental representation, with

$$
\left[t_{a}, t_{b}\right]=i f_{a b c} t_{c}, \quad \operatorname{Tr}\left(t_{a} t_{b}\right)=\frac{1}{2} \delta_{a b}
$$

$f_{a b c}$ are the structure constants of $S U(k+1)$ in this basis. (We take what is the conjugate of the fundamental representation in the conventional sense. For example, for $S U(3)$, our matrices are given by $t_{a}=-\frac{1}{2} \lambda_{a}^{T}$, where $\lambda_{a}$ are the standard Gell-Mann matrices and the superscript $T$ denotes the transpose.) We also define the differential operators $L_{a}, R_{a}$ on $g$ corresponding to the left and right translations as

$$
L_{a} g=t_{a} g, \quad R_{a} g=g t_{a}
$$

It is convenient to split these into the $R_{k^{2}+2 k}$ which is the $U(1)$ generator in $U(k) \subset S U(k+1)$, $R_{j}, j=1,2, \cdots, k^{2}-1$, which are $S U(k)$ generators and $R_{ \pm i}, i=1,2, \cdots, k$ which are in the complement of $\underline{U(k)}$ in the Lie algebra $\underline{S U(k+1)}$.

For our purpose, it is sufficient to consider a background magnetic field which is valued in $\underline{U(1)}$. The fact that we have a $U(1)$ background field can be expressed on the wave functions as [10]

$$
\begin{aligned}
R_{j} \Psi(g) & =0, \quad j=1, \cdots, k^{2}-1 \\
R_{k^{2}+2 k} \Psi(g) & =n k \frac{1}{\sqrt{2 k(k+1)}} \Psi(g)
\end{aligned}
$$

where $n$ is an integer characterizing the strength of the field. For the lowest Landau level, we also have

$$
R_{+i} \Psi(g)=0
$$


The wave functions obeying the conditions (5) and (6) are given by

$$
\Psi_{m}(g)=\sqrt{N} \mathcal{D}_{m, n}^{(n)}(g)
$$

where the Wigner $\mathcal{D}$-functions of $S U(k+1)$ are defined as

$$
\mathcal{D}_{p q}^{(n)}(g) \equiv\langle n, p|\hat{g}| n, q\rangle
$$

They are the representatives of $g$ in the rank $n$ symmetric representation; the right index on $\mathcal{D}_{m, n}^{(n)}$ occurring in (17) is fixed to be $n, \mathcal{D}_{m, n}^{(n)}(g)=\langle n, m|\hat{g}| n, n\rangle$. Also, $N$ is the dimension of the representation, $N=(n+k) ! / n ! k !$.

The large $n$ expansion is most easily obtained using the symbol of a matrix defined by

$$
(\hat{A})=A(g)=\sum_{m s} \mathcal{D}_{m, n}^{(n) *}(g) A_{m s} \mathcal{D}_{s, n}^{(n)}(g)=\left\langle w\left|\hat{g}^{-1} \hat{A} \hat{g}\right| w\right\rangle
$$

The symbol of a matrix $\hat{A}$ coincides with the expectation value of $\hat{A}$ in the large $n$ limit. Further, the trace of a matrix is given in terms of its symbol by

$$
\operatorname{Tr} \hat{A}=\sum_{m} A_{m m}=N \int d \mu(g) A(g)
$$

where the integration is over the phase space, $\mathbf{C P}^{k}$ in our case. The symbol corresponding to the product of two matrices is given by the star product of the symbols of the matrices. It can be written as

$$
(\hat{A} \hat{B}) \equiv(\hat{A}) *(\hat{B})=A B-\frac{1}{n} \hat{R}_{-i} A \hat{R}_{+i} B+\mathcal{O}\left(1 / n^{2}\right)
$$

We will not need the full expansion for the calculations presented here. Equation (10) and (11) tell us that it is possible to replace the trace of a product of matrices by the integral of the star product of the symbols of the matrices. In the star product, the leading term is just the product of the symbols, with corrections which involve derivatives, these being subdominant at large $n$.

Fuzzy $\mathbf{C P}^{k}$ can also be described, before we take the large $n$ limit, in a way which reproduces its embedding in $\mathbf{R}^{k^{2}+2 k}$ when $n$ becomes large. In this case, $\mathbf{C P}_{F}^{k}$ is given by $k^{2}+2 k$ hermitian matrices $X_{a}$ which are of dimension $(N \times N)$. The embedding conditions are then given by 14

$$
\begin{aligned}
X_{a} X_{a} & =\frac{n k(n+k+1)}{2(k+1)} \equiv C_{n} \\
d_{a b c} X_{b} X_{c} & =(k-1) \frac{(2 n+k+1)}{4(k+1)} X_{a} \equiv \alpha_{n} X_{a}
\end{aligned}
$$


where $C_{n}$ is the quadratic Casimir operator and $\alpha_{n}$ is another invariant related to the properties of the $d_{a b c}$-symbol. These embedding equations are solved by $X_{a}=T_{a}$, where $T_{a}$ are the $S U(k+1)$-generators in the symmetric representation of rank $n$. The generators $-i T_{a}$ also play the role of derivatives via their adjoint action. On the fuzzy space, gauge fields are described by $D_{a}=-i T_{a}+A_{a}$, where $A_{a}$ is the gauge potential. It is still a matrix at this stage. A priori there are $k^{2}+2 k$ spatial components for the gauge potential, but there are restrictions on $D_{a}$ which ensure that there are only $2 k$ spatial components for the potentials, as expected for $\mathbf{C P}^{k}$. These conditions are the gauged version of the conditions (12),

$$
\begin{aligned}
D_{a} D_{a} & =-C_{n} \\
d_{a b c} D_{b} D_{c} & =-i \alpha_{n} D_{a}
\end{aligned}
$$

Thus, even after the gauging $-i T_{a} \rightarrow-i T_{a}+A_{a}$, the derivatives obey the same embedding conditions (12) as before gauging [15].

The role of the gauge field is further clarified by the following identities which are easily proved. The symbol for the product of $T_{a}$ with a matrix $\hat{A}$ is given by

$$
\begin{aligned}
& \left(T_{a} \hat{A}\right)=\left[\frac{n k}{\sqrt{2 k(k+1)}} S_{a k^{2}+2 k}-\frac{1}{2} \sum^{k} S_{a-i} R_{+i}\right] A(g) \\
& \left(\hat{A} T_{a}\right)=\left[\frac{n k}{\sqrt{2 k(k+1)}} S_{a k^{2}+2 k}+\frac{1}{2} \sum^{k} S_{a+i} R_{-i}\right] A(g)
\end{aligned}
$$

where $S_{a b}=2 \operatorname{Tr}\left(g^{-1} t_{a} g t_{b}\right)$. If conditions (13) are satisfied, then the symbol of $A_{a}$ is of the form $S_{a i} A_{i}$. Equations (14) then show that the commutator is equivalent to the derivative $R_{i}$, and also that, upon gauging, we get $R_{i} \rightarrow R_{i}+A_{i}$.

Now, if $K$ is a function on fuzzy $\mathbf{C P}^{k}$, by using the symbol, we can obtain its large $n$ expansion. We can think of $K$ as given in terms of a basis made of the identity matrix, $\left(-i T_{a}\right)$ and sums of products of ( $-i T)$ 's, suitably orthonormalized. In the large $n$ expansion, the $T$ 's get replaced by $S_{a k^{2}+2 k}$ and $S_{a i} R_{i}$, as in equations (14). This corresponds to expansion with only a background $U(1)$-field for $\mathbf{C P}^{k}$ as in (5). We can also expand the same matrix $K$ in terms of a basis made of $D$ 's rather than $(-i T)$ 's. This will correspond to the $\mathbf{C P}^{k}$ having the gauge field $A_{i}$ in addition to the $U(1)$-field.

To carry out these expansions, we write $A_{0}, A_{i}$ in terms of $(N \times N)$-blocks. In other words, we write the matrix elements of $A_{i}, i=0,1,2, \ldots$ as $A_{i A B}=\left\langle A\left|A_{i}\right| B\right\rangle=\left\langle\alpha a\left|A_{i}\right| \beta b\right\rangle$, $\alpha, \beta=1,2, \cdots, N, a, b=1,2, \cdots, M$, corresponding to the Hilbert space $\mathcal{H}_{\mathcal{N}}$ being a tensor product $\mathcal{H}_{N} \otimes \mathcal{H}_{M} . \mathcal{H}_{N}$ will carry an irreducible representation of $S U(k+1)$, specifically the symmetric rank $n$ representation. In carrying out a large $n$ expansion, we will be rewriting 
the traces in terms of integrals over (the star products of) the symbols as in (10). The symbols corresponding to a matrix in this direct product splitting is defined by

$$
\left(A_{i}\right)_{a b}=\sum_{\alpha, \beta} \mathcal{D}_{\alpha, n}^{(n) *}(g)\left\langle\alpha a\left|A_{i}\right| \beta \quad b\right\rangle \mathcal{D}_{\beta, n}^{(n)}(g)
$$

The symbol $\left(A_{i}\right)_{a b}$ is thus a matrix-valued function; it may be taken to be in the Lie algebra of $U(M)$. The trace of a matrix on $\mathcal{H}_{\mathcal{N}}$ then gets converted to the integral of the symbol over the manifold with a remaining trace over $\mathcal{H}_{M}$.

As mentioned at the beginning of this section, for a group coset manifold such as $\mathbf{C P}^{k}=$ $S U(k+1) / U(k)$, it is also possible to choose the background field to be nonabelian, valued in the Lie algebra of $U(k)$ [10. For such a case, the wave functions are of the form $\mathcal{D}_{A a}^{(J)}(g)$, where $J$ denotes a representation of $S U(k+1)$, and we also have a nontrivial representation of $S U(k) \in U(k)$ for the right translations of $g$, corresponding to the index $a$, in addition to the nontrivial action of $R_{k^{2}+2 k}$. The definition of the symbol then becomes

$$
\left(A_{i}\right)_{a b}=\sum_{A, B} \mathcal{D}_{A, a}^{(J) *}(g)\left\langle A\left|A_{i}\right| B\right\rangle \mathcal{D}_{B b}^{(J)}(g)
$$

This definition, which was used in [10] and [16], is a little bit different from (15). We shall use (15) in what follows. The resulting expressions will involve functions which are also matrices in $\mathcal{H}_{M}$. If $U(M)$ contains $S U(k+1)$ as a subgroup, then, at this stage, we can introduce additional $\mathcal{D}$ 's using $\delta_{a b}=\mathcal{D}_{a c}^{K *} \mathcal{D}_{b c}^{K}$, where these $\mathcal{D}^{K}$ 's are in a suitable representation (or representations) of $S U(k+1)$ and reduce the product $\mathcal{D}_{\beta, n}^{(n)} \mathcal{D}_{b c}^{K}$ to get wave functions like $\mathcal{D}_{B c}^{(J)}$. Results using (16) can thus be recovered from results obtained using (15). Notice that a part of the remaining gauge group $U(M)$, namely $S U(k+1)$, is the isometry group of the space, and so, this procedure can be viewed as gauging the isometry group, the indices $a, b$ in (15) taking the place of the tangent space indices.

We now introduce the notation $\left[D_{a}, D_{b}\right]=f_{a b c} D_{c}+F_{a b} \equiv \Omega_{a b}$, which is the definition of the field strength $F_{a b}$. The function $K$ can be taken as the sum of terms of the form $K=K^{a_{1} a_{2} \cdots a_{s}} D_{a_{1}} D_{a_{2}} \cdots D_{a_{s}}$, where the coefficients $K^{a_{1} a_{2} \cdots a_{s}}$ can be taken to be symmetric in all indices. (Any antisymmetric pair may be reduced to a single $D$ and $F ; F$ itself may be re-expanded in terms of $D$ 's, to bring it to this form.) Thus $K$ has the form

$$
K=\int d \mu e^{\bar{z} \cdot D} K(z)
$$

where

$$
d \mu=\prod_{a} \frac{d z_{a} d \bar{z}_{a}}{\pi} e^{-\bar{z} \cdot z}, \quad K(z)=K^{a_{1} a_{2} \cdots a_{s}} z_{a_{1}} z_{a_{2}} \cdots z_{a_{s}}
$$

We now want to express $K$ in an expansion around a perturbed version of the $D$ 's, namely, $D_{a}^{\prime}$ where $A_{a}^{\prime}=A_{a}+\delta A_{a}$. Clearly this can be achieved by writing $D_{a}=D_{a}^{\prime}-\delta A_{a}=D_{a}^{\prime}-\delta D_{a}$ 
in the expression for $K$. The change in $K$ is thus given by $D_{a} \rightarrow D_{a}-\delta D_{a}$. Varying the expression (17) and taking traces, we find

$$
\begin{aligned}
\operatorname{Tr} \delta K & =-\operatorname{Tr}\left(\delta D_{a} K^{a}\right) \\
K^{a} & =\int d \mu \bar{z}^{a} e^{\bar{z} \cdot D} K(z)=\int d \mu e^{\bar{z} \cdot D} \frac{\partial K}{\partial z_{a}}
\end{aligned}
$$

Again using (17), we find

$$
\begin{aligned}
{\left[D_{a}, K\right] } & =\left[D_{a}, D_{b}\right] K^{b}-\frac{1}{2}\left[\left[D_{a}, D_{b}\right], D_{c}\right] K^{b c}+\cdots \\
& =K^{b}\left[D_{a}, D_{b}\right]+\frac{1}{2} K^{b c}\left[\left[D_{a}, D_{b}\right], D_{c}\right]+\cdots \\
K^{b c} & =\int d \mu \bar{z}^{b} \bar{z}^{c} e^{\bar{z} \cdot D} K(z)
\end{aligned}
$$

We can define a quantity $N_{a b}$ by the formula 12

$$
\begin{aligned}
N_{a c} \Omega_{c b} & =\delta_{a b}+\mathbb{X}_{a b}+i \mathbb{Y}_{a b} \\
\Omega_{b c} N_{c a} & =\delta_{b a}+\mathbb{X}_{b a}+i \mathbb{Y}_{b a}
\end{aligned}
$$

where

$$
\mathbb{X}_{a b}=\frac{D_{b} D_{a}}{B_{n}}, \quad \mathbb{Y}_{a b}=\frac{1}{B_{n}}\left(n+\frac{1}{2}(k+1)\right)\left(d_{a b c} D_{c}+i \frac{\alpha_{n}}{2} \delta_{a b}\right)
$$

$\Omega_{a b}$ does not have an inverse, but $N_{a b}$ serves the purpose when applied on quantities which obey the embedding conditions (13). $N_{a b}$ has the series solution

$$
\begin{aligned}
N_{a b} & =N_{0 a b}-\left(\mathbb{R}_{a b} N_{0}\right)_{a b}-\left(N_{0} F N_{0}\right)_{a b}+\cdots \\
N_{0 a c} & =\frac{1}{B_{n}}\left[f_{a c k} D_{k}+\frac{1}{4}(k-1) \delta_{a c}\right] \\
B_{n} & =\frac{n(n+k+1)}{4}+\frac{k^{2}-1}{16}
\end{aligned}
$$

We can now simplify equation (19) as

$$
\begin{aligned}
& \operatorname{Tr}(\delta K)=-\frac{1}{2} \operatorname{Tr}\left[\delta D_{a} N_{a b}\left(\Omega_{b c} K^{c}\right)-\delta D_{a}\left(K^{c} \Omega_{b c}\right) N_{b a}\right. \\
&\left.+\frac{1}{B_{n}}\left[\delta D_{a}\left(\Omega_{a b} K^{b}\right)-\delta D_{a}\left(K^{b} \Omega_{a b}\right)\right]\right] \\
&=-\frac{1}{2} \operatorname{Tr}\left[\delta D_{a} N_{a b}\left[D_{b}, K\right]-\left[D_{b}, K\right] N_{b a} \delta D_{a}+\mathcal{O}\left(1 / n^{3}\right)\right]
\end{aligned}
$$

where we have used equation (20). What is needed now is to write this in terms of symbols and simplify it. This is a fairly long calculation, but eventually leads to the following result. More details of this calculation can be found in [12]. 
The action of interest is the analog of (2), with $U=1$. This is the one-dmensional Chern-Simons action $S=i \int d t \operatorname{Tr}\left(D_{0}\right)$. This is simplified as

$$
i \int d t \operatorname{Tr}\left(D_{0}\right) \approx S_{* C S}+\cdots
$$

where $S_{C S}$ is the Chern-Simons $(2 k+1)$-form on $\mathbf{C P}^{k} \times \mathbf{R}$. This is defined by the variation

$$
\delta S_{C S}=\frac{i^{k+1}}{(2 \pi)^{k} k !} \int \operatorname{tr}\left(\delta A F^{k}\right)
$$

$S_{* C S}$ in (25) is the star-version of the same action, i.e., $S_{C S}$ with star products connecting the various fields in it. The gauge potentials in the Chern-Simons action are given by $a+A$, where $a$ is the background value corresponding to the symplectic form and $A$ is the additional potential or gauge field fluctuation. If we take the gradients of the fields to be small compared to their values, so that $\left|D^{2} F\right| \ll|F F|$, for example, then the higher terms in the star product are negligible and we can write 12 .

$$
i \int d t \operatorname{Tr}\left(D_{0}\right) \approx S_{C S}(a+A)+\cdots
$$

This is our basic result. Notice that only the combination $a+A$ appears in the action; thus the dependence on the specific choice of the $\mathbf{C P}^{k}$ background has disappeared. Different choices of the background potentials correspond to different large $n$ limits. Of course, the action is also sensitive to the dimension; the fact that we chose to expand in terms of a $2 k$ dimensional phase space shows up in equation (27). The large limits are thus parametrized by the gauge potentials and the choice of the dimension.

The action (27) is also the action which arises for the lowest Landau level for different gauge field backgrounds [16]. Thus it is the action for the bulk dynamics for the quantum Hall system. We will now go back to the question of how this applies to gravity on a fuzzy space.

\section{A matrix version of gravity}

Gauge fields in the case of quantum Hall effect take values in the Lie algebra of $U(k)$. From the matrix model point of view, where we write matrices in terms of the $\mathcal{H}_{N} \otimes \mathcal{H}_{M}$ splitting, there is no obstruction to extending this to $S U(k+1)$ or even any unitary group. (If we allow all possible types of fluctuations, it is a unitary group $U(M)$ for some $M$, rather than $S U(M)$, that is relevant. This is also what is needed on noncommutative spaces.) At this stage, it is worth recalling that ordinary Minkowski space may be considered as the coset space $P / L$, where $P$ is the Poincaré group and $L$ is the Lorentz group. For the case of 
$\mathbf{C P}^{k}=S U(k+1) / U(k)$, the group $S U(k+1)$ is the analog of the Poincaré group and the isotropy group $U(k)$ is he analog of the Lorentz group. The gauge fields we have introduced correspond to the gauging of these groups which are isometries of $\mathbf{C P}^{k}$, implying that they should be interpreted as gravitational fields. Based on the idea that the states which describe space itself should be treated exactly as the states for matter are treated, we see that the simplification of the action in the previous section can be used.

There are, evidently, some missing ingredients which have to be taken care of before this can be interpreted in terms of gravity. First of all, the gauge fields are of the form $A_{\mu}^{a} d x^{\mu}\left(-i t^{a}\right)$, which are one-forms on $\mathbf{C P}^{k} \times \mathbf{R}$ (because $d t$ is included in this expression) and the Lie algebra matrices form a basis for $U(k+1)$, or some other unitary group. There is, so far, no analog of $e_{\mu}^{0} d x^{\mu}$ or $\omega_{\mu}^{0 a} d x^{\mu}$, corresponding to the time-components of the frame field or spin connection. Secondly, there is a dimensional mismatch since $U(k+1)$ gives $(k+1)^{2}$ one-form fields on a $(2 k+1)$-dimensional space, where as we we need $(2 k+1)(k+1)$ one-forms to describe gravity. Thirdly, the matrix traces are naturally positive and lead to Euclidean signature for the tangent space. We shall now see how these issues can be addressed.

We start again with a finite-dimensional Hilbert space $\mathcal{H}$ describing the possible states of a quantum system. At this stage we do not even have a notion of time, but just this Hilbert space $\mathcal{H}$ and, let us say, the system under study is observed to be in a state given by a density matrix $\rho_{0}$. Any unitary transformation of $\rho_{0}$ is also an allowed density matrix, therefore, what constitutes a change in the system is a unitary transformation. In other words, we can describe trajectories in $\mathcal{H}$ which correspond to sequences of unitary transformations. The notion of evolution of the system arises when we are able to compare the density matrix with the density matrix of another reference system. Changes in the density matrix can be considered as a function of a sequence of changes in the reference system and this is the meaning of time-evolution. Let $t$ denote the parameter along the trajectory of $\rho_{0}$ in $\mathcal{H}$. The motion along the trajectory is generated by some hermitian operator $K$ defined by $i \dot{U}=K U$. (The parameter $t$ is not necessarily time, it is just the parameter along the trajectory. Later, after a large $N$ limit is taken, it may be identified with time.) The density matrix $\rho \equiv U \rho_{0} U^{\dagger}$ obeys the equation

$$
i \partial_{0} \rho=i \frac{\partial \rho}{\partial t}=[K, \rho]
$$

This equation is essentially the definition of $K$. However, if $K$ is given, it can be taken as defining the evolution of $\rho$. The action which leads to this equation is given by

$$
S=i \int d t \operatorname{Tr}\left[\rho_{0} U^{\dagger}\left(\partial_{0}+A_{0}\right) U\right] \equiv i \int d t \operatorname{Tr}\left[\rho_{0} U^{\dagger} D_{0} U\right]
$$

where $A_{\tau}=i K$. Notice also that this action (29) has a natural gauge invariance, $U \rightarrow h U$, $A_{0} \rightarrow h A_{0} h^{\dagger}-\partial_{0} h h^{\dagger}$. The action (29) is a function of $U$ and gives equation (28) as the 
variational equation $\delta S=0$ for variations of $U$.

We now consider a separation of this quantum system into a part corresponding to the degrees of freedom of space and a part which describes all other, material, degrees of freedom, denoted as the subsystem $S$. In other words, $\mathcal{H}=\mathcal{H}_{\mathcal{N}} \otimes \mathcal{H}_{S}$. Correspondingly, a state in the Hilbert space may be represented as $|A, r\rangle$. The labels $A, B$, etc., pertain to the degrees of freedom of space (or 'environment') and the labels $r, s$, etc., describe the subsystem of interest. For the operator $D_{0}$, we introduce the splitting

$$
\left\langle A, r\left|D_{0}\right| B, s\right\rangle=\delta_{r s}\left\langle A\left|D_{0}^{(e)}\right| B\right\rangle+\left\langle A, r\left|D_{0}^{(s)}\right| B, s\right\rangle
$$

The part of $D_{0}$ which is proportional to the identity in $\mathcal{H}_{S}$ is designated as $D_{0}^{(e)}$ and the remainder as $D_{0}^{(s)}$. The latter includes effects of coupling the subsystem of interest to the spatial degrees of freedom. The density matrix also has a splitting of the form

$$
\left\langle A, r\left|\rho_{0}\right| B, s\right\rangle=\delta_{A B}\left\langle r\left|\rho_{0}\right| s\right\rangle
$$

(This is not normalized, normalization will be taken care of later.) The rank of the density matrix is a measure of how much of the Hilbert space is covered by the chosen state of the system. If its rank is less than maximal for $\mathcal{H}_{\mathcal{N}}$, it would mean that the dynamics does not cover all of space. This is why we choose it to be of maximal rank in $\mathcal{H}_{\mathcal{N}}$.

Our proposal for fuzzy gravity is then the following. We take the action (29) as the action for the theory, including gravity, where $U$ and $D_{0}^{(e)}$ are regarded as quantities to be varied. $D_{0}^{(s)}$ is to be regarded as a given operator, specifying the subsystem of interest.

The notion of continuous space emerges in the limit of the dimension of $\mathcal{H}_{\mathcal{N}}$ becoming large. One may regard $\mathcal{H}_{\mathcal{N}}$ as arising from the quantization of some phase space $\mathcal{M}$, with an appropriate symplectic form. The background fields on this phase space can be varied. Thus it is possible to calculate the action, expanding $D_{0}^{(e)}$ in terms of the background gauge fields, in the limit of the dimension of $\mathcal{H}_{\mathcal{N}}$ becoming large. This is, of course, what we have done in the previous section. The best background to expand around is then given by the extremization of the action. Notice that the choice of spatial geometry in terms of these background fields arises as a choice of which large $\mathcal{N}$ limit is best suited for the study of the subsystem of interest. In this sense, the extremization with respect to these variables is more like the choice of a thermodynamic state rather than a choice of dynamical trajectory. The extremization with respect to $U$, with $D_{0}^{(s)}$ specifying the subsystem of interest is the usual, dynamical, choice of a trajectory.

We now consider this idea in more detail. The action can be written as

$$
S=i \int d t \sum_{A, r, s}\left(\rho_{0}\right)_{r s}\left\langle A, s\left|U^{\dagger} D_{0} U\right| A, r\right\rangle
$$


If the interaction between the material system and the spatial (gravitational) degrees of freedom is small, then, as a first approximation, we can look at the action for the spatial degrees of freedom by itself. Thus

$$
S \approx i \int d t \operatorname{Tr}\left(D_{0}^{(e)}\right)
$$

For expansion around $\mathbf{C P}^{k}$, we can simplify this, in the large $n$ limit and for slowly fields, to obtain the Chern-Simons action $S_{C S}$ with the gauge fields being $a+A$. Notice that this depends only on the full gauge field, the separation into $a$ and the fluctuation $A$ is immaterial. In the following, we shall rename the combination $a+A$ as $A$. We now see the basic consequence of our assumption that the spatial degrees of freedom in $\mathcal{H}_{\mathcal{N}}$ should be treated in the same way as the dynamical degrees of freedom in $\mathcal{H}_{S}$. It implies that the gravitational action, which determines the best geometry on which further analysis of the matter dynamics can be carried out, should be the Chern-Simons action [17. In particular, this will work only for odd dimensional spacetimes. We also note that there are indications of Chern-Simons gravity in the context of M-theory [18]; we expect that our approach is related to a matrix version of some of the considerations in these references.

The gauge group which occurs in the CS action should be a unitary group, say, $U(M)$, since it arises from the splitting $\mathcal{H}_{\mathcal{N}}=\mathcal{H}_{N} \otimes \mathcal{H}_{M}$, with $A_{A B}=\langle A|A| B\rangle=\langle\alpha a|A| \beta b\rangle$, $\alpha, \beta=1,2, \cdots, N, a, b=1,2, \cdots, M$. While we interpret $\mathcal{H}_{N}$ as the quantization of $\mathbf{C P}^{k}$ with the symplectic form $\omega=-i n \omega_{K}$, a priori, there is no restriction on $M$, we could choose any unitary group. However, since $\mathbf{C P}^{k}=S U(k+1) / U(k)$, the possible gauge field fluctuations which can be interpreted as gravity belong to $S U(k+1)$, or at best, $U(k+1)$. Therefore, we take $M=k+1$.

The question of the dimensional mismatch can be handled by using an idea similar to compactification. The simplest case for which this can be carried out is for $k=3$, corresponding to the group $U(4)$. The Chern-Simons action is thus defined on a seven-dimensional space. We take this to be of the form $S^{2} \times M^{5}$, where $M^{5}$ is some five-dimensional manifold. Writing $U(4) \sim S U(4) \times U(1)$, the gauge field is taken to be of the form $-i l \omega_{K}+\mathcal{F}$, where $\omega_{K}$ is the Kähler form of the two-sphere $S^{2}, l$ is an integer and $\mathcal{F}$ belongs to the $S U(4)$ Lie algebra. The effective action is then given by the level $l$, five-dimensional Chern-Simons action with the gauge group $S U(4)$,

$$
S=-i \frac{l}{24 \pi^{2}} \int \operatorname{tr}\left(A d A d A+\frac{3}{2} A^{3} d A+\frac{3}{5} A^{5}\right)
$$

Since $S U(4)$ is locally isomorphic to $O(6)$, we see that we have the correct set of gauge fields to describe Euclidean gravity in five dimensions. In fact, we can expand the gauge potential as

$$
A=P^{a} e_{\mu}^{a} d x^{\mu}+\frac{1}{2} J^{a b} \omega_{\mu}^{a b} d x^{\mu}
$$


where $J_{a b}$ are the generators of $O(5) \subset O(6)$ and $P_{a}$ are a basis for the complement of $\underline{O(5)}$ in $O(6)$. As a specific matrix representation, we take $P_{a}=-(i / 2) \gamma_{a}, J_{a b}=(1 / 4)\left[\gamma_{a}, \gamma_{b}\right], \gamma^{\prime}$ s being the four-dimensional Dirac matrices. In equation (35), $e^{a}$ can be identified as the frame fields and $\omega^{a b}$ as the spin connection. The variation of the action (34) can now be simplified as

$$
\delta S=-\frac{l}{128 \pi^{2}} \int\left[\delta \omega^{a b} \mathcal{R}^{c d}(\mathcal{D} e)^{e}+\frac{1}{2} \delta e^{a} \mathcal{R}^{b c} \mathcal{R}^{d e}\right] \epsilon_{\text {abcde }}
$$

where

$$
\begin{aligned}
(\mathcal{D} e)^{a} & =d e^{a}+\omega^{a c} e^{c} \\
\mathcal{R}^{a b} & =R^{a b}-e^{a} e^{b} \\
R^{a b} & =d \omega^{a b}+\omega^{a c} \omega^{c b}
\end{aligned}
$$

We see that $R^{a b}$ is the Riemann tensor for the spin connection and $(\mathcal{D} e)^{a}$ is the torsion tensor. The equations of motion for gravity with no matter field are then

$$
\begin{aligned}
\epsilon_{a b c d e} \mathcal{R}^{c d}(\mathcal{D} e)^{e} & =0 \\
\epsilon_{a b c d e} \mathcal{R}^{b c} \mathcal{R}^{d e} & =0
\end{aligned}
$$

The solution to these equations, corresponding to empty space with no matter, is thus given by

$$
A=g^{-1} d g, \quad g \in O(6)
$$

This space is $O(6) / O(5)=S^{5}$ which is the Euclidean version of de Sitter space. It is given in a basis where the cosmological constant has been scaled out; it may be introduced by the replacement $e^{a} \rightarrow \sqrt{\Lambda} e^{a}$. The full solution of the CS action on the seven-dimensional space is thus $S^{2} \times S^{5}$, where the $U(1)$ component has the nonzero value $-i l \omega_{K}$ on the $S^{2}$ and the $O(6)$ fields have the nonzero value given by (39) on the $S^{5}$.

There is also a neat reduction of this to four dimensions [19. Going back to the fivedimensional theory (34), we can take $e_{5}^{5}=1, \omega^{5 a}=0, \omega_{5}^{a b}=0$, for $a, b=1, \ldots, 4$. The fifth dimension is taken as a circle of, say, unit radius. In this case, (34) gives

$$
\begin{aligned}
\delta S & =-\frac{l}{32 \pi} \int\left[\delta \omega^{a b} e^{c}(\mathcal{D} e)^{d}-\delta e^{a} e^{b} \mathcal{R}^{c d}\right] \epsilon_{a b c d} \\
& =\delta\left[\frac{l}{64 \pi} \int\left(e^{a} e^{b} R^{c d}-\frac{1}{2} e^{a} e^{b} e^{c} e^{d}\right) \epsilon_{a b c d}\right]
\end{aligned}
$$

We see that the resulting four-dimensional action is

$$
S=\frac{l \Lambda}{64 \pi} \int\left(e^{a} e^{b} R^{c d}-\frac{1}{2} e^{a} e^{b} e^{c} e^{d}\right)
$$


(We have also done the scaling $e^{a} \rightarrow \sqrt{\Lambda} e^{a}$.) This action leads to the zero torsion condition and the vacuum Einstein equations with a cosmological constant. It can also be written in the Einstein-Hilbert form

$$
S=\frac{l \Lambda}{16 \pi} \int \sqrt{g} d^{4} x(R-3 \Lambda)
$$

The choice of the conditions $e_{5}^{5}=1, \omega^{5 a}=0, \omega_{5}^{a b}=0$ makes the theory into a nontopological theory.

The case of other dimensions can be treated similarly. For example, we could go back to the five-dimensinal case (34) and introduce a compactification to three dimensions by choosing $M^{5}=S^{2} \times M^{3}$ and picking a background value for $d e^{5}$ as the Kähler two-form on the $S^{2}$. Upon integrating over this $S^{2}$, we will get a three-dimensional CS form with the gauge group $O(4)$. This can be interpreted as the action for three-dimensional gravity as in 20].

One can also start in higher dimensions. For example, if we start with 11 dimensions, which would correspond to a group $U(6)$, we can choose $M^{11}$ as $\mathbf{C P}^{2} \times M^{7}$, where the $\mathbf{C P}^{2}$ space has a background which is $U(2)$-valued. This gives a reduction to seven dimensions with $U(4)$ fields on it, and further reduction can be done as before. However, to get fullfledged gravity in 11 dimensions, with $O(12)$-valued gauge fields, we will need to start with the expansion of the CS one-form matrix action (33) corresponding to a higher dimensional space which has aunitary group which can accommodate $O(12)$.

We shall now comment briefly on the issue of Minkowski signature. The action involves the trace over the matrix labels of $U(M)$. For the five-dimensional theory, this corresponds to the spinor representation of $O(6)$, which is also the fundamental representation of $S U(4)$. This is constructed in terms of the $\gamma$-matrices which obey $\gamma_{a} \gamma_{b}+\gamma_{b} \gamma_{a}=2 \delta_{a b}, a, b=1, \ldots, 5$. We can rewrite the generators of $S U(4)$ in terms of a new set of $\gamma$-matrices, say, $\tilde{\gamma}_{a}=\gamma_{a} \gamma_{4}$, $a \neq 4, \tilde{\gamma}_{0}=\gamma_{4}$. This leads to $\tilde{\gamma}_{a} \tilde{\gamma}_{b}+\tilde{\gamma}_{b} \tilde{\gamma}_{a}=2 \eta_{a b}$, where $\eta_{a b}$ is the Minkowski metric. We can now expand the fields $A$ in terms of $\tilde{\gamma}_{a}, \tilde{\gamma}_{a b}$, identifying the components as $O(5,1)$ gauge fields. This defines a particular Minkowski continuation. While this can formally carry out a continuation of the tangent frame indices, how the proper continuation can be done for the matter part is not yet clear. We hope to address this question in more detail in a future publication.

I thank Dimitra Karabali for useful discussions. This work was supported in part by the National Science Foundation grant number PHY-0244873 and by a PSC-CUNY grant. 


\section{References}

[1] A. Connes, Nocommutative Geometry (Academic Press, 1994); J. Madore, An Introduction to Noncommutative Geometry and its Physical Applications, LMS Lecture Notes 206 (1995); G. Landi, An Introduction to Noncommutative Spaces and their Geometry, Lecture Notes in Physics, Monographs m51 (Springer-Verlag, 1997).

[2] For a review of the matrix version of M-theory and its solutions, see, W. Taylor IV, Rev. Mod. Phys. 73 (2001) 419; for a review of field theories on noncommutative spaces, see, M.R. Douglas and N.A. Nekrasov, Rev. Mod. Phys. 73 (2001) 977.

[3] For a recent review of fuzzy spaces and theories defined on them, see, A.P. Balachandran, S. Kurkcuoglu and S. Vaidya, hep-th/0511114.

[4] A.P. Balachandran, T.R. Govindarajan and B. Ydri, Mod. Phys. Lett. A 15 (2000) 1279; The fermion doubling problem and noncommutative geometry. II, hep-th/0006216.

[5] There are many papers on this; the paper D. Berenstein, J.M. Maldacena and H. Nastase, JHEP 0204 (2002) 013 and citations to it can give a perspective on matrices and branes.

[6] A. Chamseddine, G. Felder and J. Fröhlich, Commun. Math. Phys. 155 (1993) 205; W. Kalau and M. Walze, J. Geom. Phys. 16 (1995) 327; D. Kastler, Commun. Math. Phys. 166 (1995) 633; A. Chamseddine and A. Connes, Commun. Math. Phys. 186 (1997) 731; A.H. Chamseddine, J. Math. Phys. 44 (2003) 2534; S. Cacciatori et al, Class. Quant. Grav.19 (2002) 4029; J. Madore, gr-qc/9709002; M. Banados, et al, hep-th/0104264 J. Moffat, hep-th/0007181, hep-th/0008089 P-M. Ho, Int. J. Mod. Phys. A12 (1997) 923; N.A. Viet and K.C. Wali, hep-th/0212062; P. Aschieri et al, hep-th/0504183 hep-th/0510059 A.P. Balachandran, T.R. Govindarajan, K.S. Gupta and S. Kurkcuoglu, hep-th/0602265.

[7] V.P. Nair, Nucl. Phys. B651 (2003) 313; Yasuhiro Abe and V.P. Nair, Phys. Rev. D68 (2003) 025002.

[8] This has been known for a while; for a recent review, see D. Karabali, V.P. Nair and R. Randjbar-Daemi, in From Fields to Strings: Circumnavigating Theoretical Physics, Ian Kogan Memorial Collection, M. Shifman, A. Vainshtein and J. Wheater (eds.), World Scientific, 2004; p. 831-876.

[9] S.C. Zhang and J.P. Hu, Science 294 (2001) 823; J.P. Hu and S.C. Zhang, cond-mat/0112432 
[10] D. Karabali and V.P. Nair, Nucl. Phys. B641 (2002) 533; Nucl. Phys. B679 (2004) 427; Nucl. Phys. B697 (2004) 513.

[11] M. Fabinger, JHEP 0205 (2002) 037; Y.X. Chen, B.Y. Hou and B.Y. Hou, Nucl. Phys. B638 (2002) 220; B.A. Bernevig, C.H. Chern, J.P. Hu, N. Toumbas and S.C. Zhang, Ann. Phys. 300 (2002) 185; Y. Kimura, Nucl. Phys. B637 (2002) 177; H. Elvang and J. Polchinski, hep-th/0209104; Y.X. Chen, hep-th/0209182; S.C. Zhang, Phys. Rev. Lett. 90 (2003) 196801; B. A. Bernevig, J.P. Hu, N. Toumbas and S.C. Zhang, Phys. Rev. Lett. 91 (2003) 236803; G. Sparling, cond-mat/0211679, B. Dolan, hep-th/0304037 G. Meng, cond-mat/0306351;B. Dolan, hep-th/0304037; G. Meng, cond-mat/0306351 S. Bellucci, P. Casteill and A. Nersessian, hep-th/0306277; V.P. Nair and S. RandjbarDaemi, Nucl. Phys. B679 (2004) 447; A.P. Polychronakos, Nucl. Phys. B705 (2005) 457; Nucl. Phys. B711 (2005) 505; A. Jellal, Nucl. Phys. B725 (2005) 554.

[12] V.P. Nair, hep-th/0605007

[13] D. Berenstein, JHEP 0407 (2004) 018; H. Lin, O. Lunin and J. Maldacena, JHEP 0410 (2004) 025.

[14] V.P. Nair and S. Randjbar-Daemi, Nucl. Phys. B 533 (1998) 333; H. Grosse and A. Strohmaier, Lett. Math. Phys. 48 (1999) 163; A.P. Balachandran et al, J. Geom. Phys. 43 (2002) 184; G. Alexanian et al J. Geom. Phys. 42 (2002) 28; G. Alexanian, A. Pinzul and A. Stern, Nucl. Phys. B 600 (2001) 531.

[15] D. Karabali, V.P. Nair and A.P. Polychronakos, Nucl. Phys. B627 (2002) 565; H. Grosse and H. Steinacker, Nucl. Phys. B707 (2005) 145.

[16] D. Karabali, Nucl. Phys. B726 (2005) 407; hep-th/0605006.

[17] For recent reviews of Chern-Simons gravity, see, J. Zanelli, Braz. J. Phys. 30 (2000) 251 and J. Zanelli, hep-th/0502193.

[18] R. Troncoso and J. Zanelli, Phys. Rev. D58 (1998) 101703; P. Horava, Phys. Rev. D59 (1999) 046004; H. Nastase, hep-th/0306269; P. Horava and C.A. Keeler, hep-th/0508024.

[19] This is related to the description of gravity developed in F. Mansouri and L. N. Chang, Phys. Rev. D13 (1976) 3192; S. MacDowell and F. Mansouri, Phys. Rev. Lett. 38 (1977) 739; F. Mansouri, Phys. Rev. D16 (1977) 2456.

[20] A.H. Chamseddine, Phys. Lett. B233 (1989) 291; Nucl. Phys. B346 (1990) 213; E. Witten, Nucl. Phys. B311 (1988) 46; A. Achucarro and P. Townsend, Phys. Lett. B180 (1986) 89. 